\title{
The Unification of Active Galaxies: Observations
}

\author{
R. W. Goodrich \\ The W. M. Keck Observatory, 65-1120 Mamalahoa Highway. Kamuela, \\ HI $96743, U S A$
}

\begin{abstract}
Unification theories have been central to much of our recent progress in the study of active galactic nuclei (AGN). These theories often explain the differences between some observationally defined classes of objects as due to what might be considered 'uninteresting' or nonfundamental parameters, such as viewing angle. In this presentation, I discuss not only such orientation-dependent unification theories, but also expand the concept to unification through other means, such as evolutionary effects.
\end{abstract}

\section{Different Flavors of Unification}

The unification of AGN has largely been observationally driven, perhaps most strongly by the demonstrations by Antonucci \& Miller (1985), Miller \& Goodrich (1990), and others that a number of Seyfert 2s, previously thought to lack the broad-line region (BLR) characteristic of Seyfert 1s, show Seyfert 1 spectra in polarized light. This led to the conclusion that Seyfert $1 \mathrm{~s}$ and $2 \mathrm{~s}$ may in fact be the same type of object, simply seen from different viewing angles. In the case of Seyfert $2 \mathrm{~s}$, our line of sight to the BLR is blocked from direct view, and we see the broad lines and much of the continuum only via scattered, polarized light.

This 'Seyfert unification' is an example of unification through orientation; in other words, classes of objects previously thought to be distinct are in fact shown to be the same, just viewed from different angles. Another well-known example is the identification of blazars and superluminal radio sources as nearly pole-on radio galaxies and quasars. Emission from the relativistic jets in these objects beams and boosts their synchrotron radiation preferentially in the polar direction, while foreshortening of the relativistic motions of radio components explains the apparently superluminal motions.

Other forms of unification are possible, however. As an example one might discuss unification in time. This is generally thought of as evolution; if one class of objects evolves into another class they might be considered to be the same, showing different characteristics depending on what age they are. As an example from entomology, you don't classify a caterpillar and the butterfly it will change into as different species, despite the fact that they look quite different!

A third possibility is unification through physical processes. At the beginning of this conference we heard a lot of interesting information about the so-called 'narrow-line Seyfert 1s' (NLS1s). The NLS1s are likely to be extreme 
examples of the Seyfert 1 population, with few fundamental physical differences. However, some NLS1s appear to have fairly unusual X-ray characteristics, and hopefully this will give us some insight into the central engines of all Seyfert 1s. When we understand what is causing these extremes of line widths and X-ray behavior, we will then have 'unified' the NLS1 and classical Seyfert 1 classes, learning some significant astrophysics in the process.

Since mine is not a review talk, I have the luxury of not having to be exhaustive in covering the topics which might fall under my purview. Since many researchers active in AGN work already know the fundamentals of the Seyfert unification story, I will skip most of that, hitting only some recent highlights which I myself find exciting. I will then update the reader on a number of other important developments away from the Seyfert story. Section $\S 3$ will discuss some examples of unification through time, and the last section will briefly discuss unification through physical processes.

\section{Unification Through Orientation - Seyfert Galaxies}

In $\S 1$, I outlined roughly the unification of Seyfert 2 s with Seyfert 1s. This is a rather 'mature' unification picture in the sense that it is fairly well established, and we are using our new understanding to delve deeper into the physics of AGN. There are a number of exciting new developments which bear generally on the unification theory.

One such development was the realization that much of the optical nonstellar continuum from Seyfert $2 \mathrm{~s}$ is actually not light reflected from hidden central regions. Hints of this came in Miller \& Goodrich (1990), but Tran's (1995) thesis drove the point home. The observational motivation behind this discovery came from the lower observed continuum polarization (after careful correction for dilution from starlight) when compared to the broad-line polarization. The measurement is difficult, relying on identifying the broad wings of scattered BLR light in the total flux, where it is nearly overwhelmed by the much brighter NLR emission. The measurement of relatively high broad-line polarizations with this technique, however, removes some of the questions raised by Miller \& Goodrich, specifically about the lack of very high-polarization Seyfert $2 \mathrm{~s}$. Tran found that the featureless continuum, however, was generally less highly polarized than the broad lines, indicating a second, unpolarized continuum source called 'FC2'.

FC2 seems to be an extended continuum which is not hidden from our direct view. Tran argues that this continuum may be generated within the scattering region itself. Curiously, since the resulting $P(\lambda)$ is more or less flat with wavelength, the scattered FC1 must have the same spectral shape as FC2, perhaps arguing for a similar source for both continua.

As detailed by other authors in this conference, the study of warm absorbers in the X-rays probably has an intimate connection to unification theories. The implied covering factors are similar to the covering factors of the obscuring torus in Seyferts, and this brings up the exciting possibility that we can start to determine physical parameters of the torus, through X-ray observations.

Another interesting report on Seyfert unification is the discovery by Nelson et al. (1996) using $H S T$ snapshots that the nuclear continuum source in Seyfert $1 \mathrm{~s}$ generally appears stellar, whereas in most Seyfert $2 \mathrm{~s}$ it appears extended on 
scales of $100-200 \mathrm{pc}$. This is the scale expected for the scattering regions of the Seyfert 2s, and opens the possibility of directly exploring the spatial structure of the scattering regions. A more detailed analysis of this data is in progress, and will give us a better handle on what new physics we will be able to learn from this.

An imaging survey of LINERs by Maoz et al. (1995) failed to find UV point sources in most of their nuclei, perhaps suggesting that in these objects, too, the central regions can be obscured by a thick torus. Spectropolarimetry of those LINERs lacking UV point sources should prove interesting!

Finally let me mention the intriguing infrared imaging of Simpson et al. (1996). Infrared images of the Seyfert 2 Mrk 348 show an oblong structure in the nucleus, extended perpendicular to the radio axis (the presumed symmetry axis). This structure could be the outer parts of the obscuring torus. Again, the possibility of spatial studies of the torus is suggested.

The HST and UKIRT work show our current ability to resolve some of the important structures in the Seyfert unification scenario. In the future, adaptive optics will further enhance our spatial resolution, with the mouth-watering possibilities of optical/IR interferometry on the distant horizon. In 1997, the new NICMOS imager on HST should provide new insight into these studies as well.

\subsection{Radio Galaxies}

Radio galaxies, like their optically quiet relatives the Seyferts, also are classified as narrow-line (NLRG) and broad-line (BLRG) objects. In analogy to the Seyfert situation, we might expect that NLRGs really are BLRGs with the BLRs and central continua hidden from our direct view. The case for this was bolstered by the discovery that the quintessential NLRG Cyg A has a hidden nuclear power source. In the optical, Tadhunter, Scarrott, \& Rolph (1990) found that the polarization vectors outside the nucleus form a vaguely centrosymmetric pattern, as expected from reflection of a point source.

However, there were a couple of nagging problems with this picture. First, Tadhunter et al. could not explain why the scattered light had such low polarization $(\sim 1 \%)$, as noted by Goodrich \& Miller (1989). At this level, the polarization correction for dust within our own Galaxy is very significant, even though it is hard to determine what that interstellar polarization might be. (In Cygnus, the Galactic polarization vectors converge, so different stars in this area can have radically different polarizations.) More damaging was the failure to find broad $\mathrm{H} \alpha$ in the polarized light of Cyg A (Jackson \& Tadhunter 1993; unpublished Keck data), although Antonucci, Hurt, \& Kinney (1994) report the possible detection of broad $\mathrm{Mg}$ II in total flux. Again, one can think of ways to save the unification picture, including the existence of a strong FC2 (which in the case of Cyg A would have to be much more extended than the FC2 that Tran invokes in Seyferts, and may include light from hot stars, as suggested by Goodrich \& Miller), the difficulty of doing spectropolarimetry on the rarer, more distant radio galaxies, and the possibility that Cyg A harbors an NLS1, and its broad lines are that much harder to distinguish from the narrow components.

Fortunately, similar recent work on other NLRGs has shown that the unification scenario is alive and well. Draper, Scarrott, \& Tadhunter (1993) showed that 3C 321 also has a centrosymmetric polarization pattern in images, this 
time uncontaminated by heavy Galactic polarization. Broad polarized lines have since been found in 3C 321 and a small number of other NLRGs (Young et al. 1996; Cohen et al., private communication), again in analogy to the Seyfert observations.

\subsection{High-Redshift Radio Galaxies}

McCarthy et al. (1987) first discussed the so-called 'alignment effect' seen in high-redshift radio galaxies. In the optical (i.e., rest-frame UV), many of these objects show emission extended along the radio axis. A number of possibilities for this effect have been discussed, including (a) jet-induced star formation, (b) reflection of a hidden QSO nucleus, with light escaping only along the polar regions, and (c) the illumination pattern of a blazar, showing how ambient gas sees more intense nuclear light when it lies along the poles.

A number of studies, well summarized by Cimatti et al. (1993), show that these high- $z$ radio galaxies are often highly polarized, a result which rules out jet-induced star formation in those objects. To determine what is being reflected requires spectropolarimetry, since explanations (b) and (c) differ mainly in whether the reflected continuum is similar to normal QSOs or blazars, i.e., shows strong emission lines or mainly continuum. Keck observations have recently confirmed hints from 4-m class instruments that there are indeed broad lines in the polarized flux (e.g. Cimatti et al. 1996), and often high $S / N$ normal spectroscopy can show broad lines (Dey \& Spinrad 1996). As these are radioloud objects, there must be some Doppler-beamed emission, and a large enough sample of high- $z$ radio galaxies may show what fraction of the alignment effect is caused by explanation (c) above.

\subsection{Broad Absorption Line QSOs (BALQs)}

Recently some new work has been initiated on the so-called BALQs. The spectra of these objects show broad, blueshifted absorption of rest-frame UV resonance lines of high-ionization species like CIV, Si IV, and NV. A minority of BALQs also show absorption in lower ionization lines like $\mathrm{Mg}$ II and $\mathrm{Al}$ III these 'lowBALs' may have qualitative differences such as dust in the BAL region (Sprayberry \& Foltz 1992). The interpretation of the BAL phenomenon is that we are seeing normal, radio-quiet QSOs from lines of sight passing through a highly ionized absorption region.

Stockman, Moore, \& Angel (1984) pointed out that a significant fraction of BALQs are highly polarized, even though almost no non-BAL radio-quiet QSOs are. Recently we pointed out that this implies that the continuum in these objects is significantly attenuated along the BAL lines of sight, at least in high- $P$ objects (Goodrich \& Miller 1995). This statement is much stronger than the observation of line absorption, since the lines have higher optical depths (by factors of $\sim 10^{4}$ and higher) than the continuum optical depths from the same clouds. Attenuation of the direct (presumably low- $P$ ) view of the central continuum source, combined with relatively unattenuated scattered (and polarized) light, produces the observed high net polarization in the BALQs. In non-BALQs, the direct view of the central source completely overwhelms the scattered light, and we see low net polarization. 
This attenuation of the optical continuum also explains the observation of Francis, Hooper, \& Impey (1993) that BALQs seem to be 'radio moderate'. By this they mean that although BALQs are always (or nearly always) radio quiet, they tend to be found on the radio-loud end of the radio-quiet distribution, as parameterized by $\log R$, where $R$ is the radio-to-optical flux ratio. Recently (Goodrich 1996) I have pointed out that this comes about because the optical continuum is attenuated by about the same amount as required to explain the difference in polarization characteristics. Hence in the BALQs, $R$ is measured to be higher than it is intrinsically.

This has further consequences! In surveys for QSOs, the majority of QSOs are found just above the flux limit. This is a consequence of the steepness of the QSO luminosity function. Assuming that all BALQs are observed to be a factor of 3-4 fainter in the optical than they would be if they were observed as nonBALQs, we can calculate how many BALQs are missed by the surveys because of this attenuation. It turns out that most are missed, and correcting for this implies that most QSOs are BALQs! In other words, the covering fraction of the BAL region is larger than $50 \%$, much higher than the $10 \%$ generally assumed from a simpler interpretation of the survey results.

Now, it is not necessary for all BALQs to be attenuated in the continuum in this interpretation, but it is necessary for all high-P BALQs to be attenuated, and as Stockman, Moore, \& Angel (1984) point out, 25\% of BALQs are highly polarized. Redoing the calculation by assuming that at least $25 \%$ of observed BALQs are attenuated gives somewhat smaller (and perhaps more comforting) numbers. The intrinsic fraction of QSOs which are BALQs is then at least $1 / 3$, still a large fraction, but more comfortably consistent with the high polarizations seen in the BALQs. Most (more than 3/4) of the BALQs are attenuated and highly polarized, although most of these are lost below the survey flux limits. A higher fraction of the low- $P$ BALQs are picked up in the surveys since they remain unattenuated.

\section{Unification Through Time}

In a word, evolution. Evolution is often difficult to study in astronomy, since we are usually presented with snapshots of different objects, some of which may be the same physical objects seen at different points in their aging process. Other snapshots will be of different objects, or objects seen from different orientations, as discussed above. Our task is to sort these into appropriate physical (as opposed to observational) classes, to allow study of true physical differences as well as the evolutionary changes which the objects undergo.

An example is the often-suggested scenario in which a recent merging event provides fuel to the central regions of a galaxy, allowing an active nucleus to turn on. In the early stages of such a merger, when the gas and dust in the system is the most highly disturbed, there may be a more or less spherical shroud surrounding the nucleus. An example is Mrk 231, which shows only a very weak, low-ionization NLR despite there being substantial amounts of surrounding gas. Either there is no low- $N_{e}$ gas in this object or the photons that would ionize the gas do not reach it. This latter interpretation implies a high covering factor for gas and dust inside the NLR. Note that IR-bright AGN 
also seem to more commonly be low-ionization BALQs than do optically selected QSOs, again consistent with a higher covering factor.

Gas clouds tend to collide with each other and dissipate energy, and a spherical distribution of clouds is expected to age by flattening into a disk. Accentuating this is the formation of strong outflows in the center of the AGN, which will tend to punch holes in the dust shroud at its weakest point. As in stellar outflows this weak point is generally along the angular momentum axis, the poles. Hence we expect that as Mrk 231 and similar objects age, the covering factor of gas and dust would decrease, a classical NLR would form, and we would see a more typical Seyfert 1 (or Seyfert 2 or QSO, depending on the orientation and luminosity). This demonstrates the idea of unification through time.

\section{1. 'Chameleons' Amongst the Seyferts}

Another example of unification through time can be sought amongst the handful of AGN which change dramatically on shorter (year- to decade-long) time scales. Such 'chameleon' Seyferts can change from classical Seyfert 1s to (or nearly to) Seyfert 2 s during a thesis student's tenure. These changes are particularly appropriate to discuss at this conference as a great deal of work on emissionline variability has already been presented. In those cases it has been assumed, generally correctly in my opinion, that the line variability is due to changes in the continuum flux, with consequent changes in the ionization state and other properties of the BLR. However, I think that I have convincingly shown (in Goodrich 1989a, 1995) that some Seyferts show emission-line (and continuum) variability due to dust clouds passing in front of the BLR. The objects which show this effect tend to be those which show the greatest degree of variability, those that change classes so dramatically.

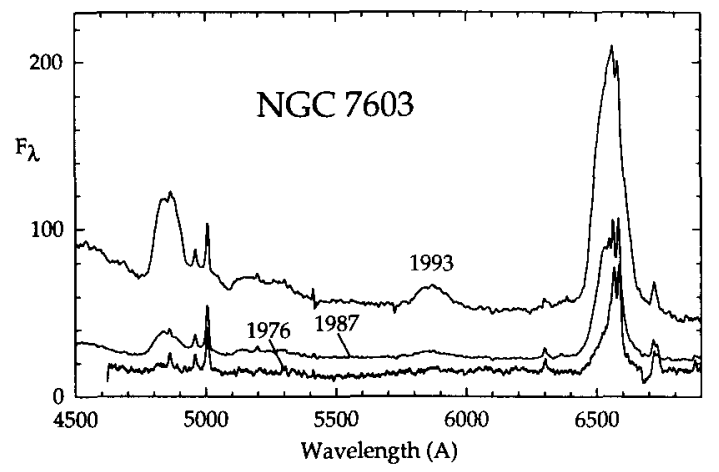

Figure 1 shows such changes in dust column towards NGC 7603. In 1976, the object was classified as a Seyfert 1.9, but since then it has become a classical Seyfert 1. If these spectral changes are due to a variable dust column, then the change in broad $\mathrm{H} \alpha$ flux gives us a measure of $\Delta E(B-V)$ (assuming a foreground dust screen covering the entire BLR, and a Galactic-type extinction law). The change in broad $\mathrm{H} \beta$ gives an independent measure of $\Delta E(B-V)$. If the variability is due to changing dust column, then these values better be the same! Agreement of the two estimates does not guarantee that the variations are due to changing $E(B-V)$; changes in the ionizing continuum may mimic 
a reddening change over some limited range in total variability. This is important; two observations drive one to the conclusion that some Seyferts are truly showing changes in dust column. One is that it becomes difficult to construct photoionization models which mimic reddening changes over the large range of variability seen in some objects. A more important observation is that the continuum and $\mathrm{He} \mathrm{I}$ emission lines also can yield similar estimates of $\Delta E(B-V)$ ! It is not obvious that the continuum should show changes consistent with the emission lines, since they may sample different parts of the dust cloud, but for the photoionization explanation it certainly adds another coincidence. When a line such as He I $\lambda 5876$, also changes in a manner consistent with reddening (as in NGC 7603), it adds yet another coincidence for the photoionization model, which at that point becomes untenable in my view. $H S T$ spectra at $\mathrm{H} \alpha$ and Ly $\alpha$ are currently testing for reddening in non-variable Seyfert 1.8/1.9s.

In the context of unification scenarios, these chameleon Seyferts then show that some time-variable effect can put a single object in one class or the other depending on when we observe it. (In a sense, these are 'self-unifying' objects.) While such short time-scale transitions are rare in astronomy, and even rarer in extragalactic astronomy in particular, we hopefully will gain a greater understanding by studying these changes. Monitoring campaigns on objects like NGC 7603 and NGC 2622 could prove very interesting, although the goal would be different from the NGC 5548-type variability studies.

\section{Unification Through Physical Processes}

Of course, our ultimate goal is always to better understand the physics in active galaxies. We want to understand how similar physical processes can manifest themselves differently in different objects. Understanding this will also help us determine when different physical processes are responsible for observed differences. In this sense if we can show that the same physical process that produces one class (or set of characteristics) can also produce a second class, we will have physically 'unified' those two classes. We can then go on to study the physical process in more clarity and detail.

An example of this might be the unification of radio-quiet QSOs, Seyfert galaxies, and perhaps LINERs. This sequence may represent a sequence in luminosity, translating into a sequence in ionization parameter, hence into different emission line characteristics. A more common example from farther afield is the spectral-type sequence, which when rearranged into the familiar OBAFGKM sequence represents decreasing surface temperature.

A second example of unification through physical processes might be the narrow-line Seyfert 1s (NLS1s). It was originally argued that NLS1s represented the extreme low end of the line-width distribution of normal Seyfert 1s, not a physically distinct class (e.g., Goodrich 1989b). Recent X-ray observations, though, have shown that some NLS1s have extremely steep soft X-ray slopes, and high X-ray variability amplitudes (e.g., Boller, Brandt, \& Fink 1996; Grupe et al. 1995). How is the low line width related to these extreme $X$-ray properties? I will not repeat the discussion of earlier authors in these proceedings, other than to hold this up as a unification process in which, hopefully, once we determine 
the physical cause for the NLS1 characteristics we will have made significant progress in understanding the physics in the central regions of all Seyfert 1s.

On an ending note, I would also like to present what I think is one of the most outstanding fundamental questions in AGN research today. That question is the nature and cause of the difference between radio-loud and radio-quiet QSOs. I will not answer that question here, but merely present it as another example of what will eventually be a unification through physical process, once we understand what that physical process is!

I would like to thank the conference organizers for hosting a very valuable and enervating conference. I would also like to thank the IAU and the AAS Travel Office for granting support for my attendance at the conference.

\section{References}

Antonucci, R., Hurt, T., \& Kinney, A. 1994, Nature, 371, 313.

Antonucci, R. R. J., \& Miller, J.S. 1985, ApJ, 297, 621.

Boller, T., Brandt, W. N., \& Fink, H. 1996, A\&A, 305, 53.

Cimatti, A., et al. 1996, ApJ, 465, 145.

Cimatti, A., et al. 1993, MNRAS, 264, 421.

Dey, A., et al. 1996, ApJ, 465, 157.

Dey, A., \& Spinrad, H. 1996, ApJ, 459, 133.

Draper, P. W., Scarrott, S. M., \& Tadhunter, C. N. 1993, MNRAS, 262, 1029.

Francis, P. J., Hooper, E. J., \& Impey, C. D. 1993, AJ, 106, 417.

Goodrich, R. W. 1989a, ApJ, 340, 190.

Goodrich, R.W. 1989b, ApJ, 342, 224.

Goodrich, R. W. 1995, ApJ, 440, 141.

Goodrich, R.W. 1996, ApJ, in press.

Goodrich, R. W., \& Miller, J.S. 1989, ApJ, 346, L21.

Goodrich, R. W., \& Miller, J.S. 1995, ApJ, 448, L73.

Grupe, D, et al. 1995, A\&A, 300, L21.

Jackson, N., \& Tadhunter, C. N. 1993, A\&A, 272, 105.

Maoz, D., et al. 1995, ApJ, 440, 91.

McCarthy, P. J. et a.l. 1987, ApJ, 321, L29.

Miller, J.S., \& Goodrich, R. W. 1990, ApJ, 355, 456.

Nelson, C. H., et al. 1996, ApJ, 466, 713.

Simpson, C., et al. 1996, ApJ, 457, L19.

Sprayberry, D., \& Foltz, C. B. 1992, ApJ, 390, 39.

Stockman, H.S., Moore, R. L., \& Angel, J. R. P. 1984, ApJ, 279, 485.

Tadhunter, C. N., Scarrott, S. M., \& Rolph, C. D. 1990, MNRAS, 246, 163.

Tran, H. D. 1995, ApJ, 440, 597.

Young, S., et al. 1996, MNRAS, 279, L72. 